Elsevier Editorial System(tm) for Mechanical Systems and Signal Processing

Manuscript Draft

Manuscript Number:

Title: ARMA Modelled Time Series Classification for Structural Health Monitoring of Civil Infrastructure

Article Type: Full Length Article

Section/Category:

Keywords: Structural Health Monitoring; Time Series Modelling; ARMA models

Corresponding Author: Dr Eoin Peter Carden, PhD

Corresponding Author's Institution: University of Sheffield

First Author: Eoin Peter Carden, PhD

Order of Authors: Eoin Peter Carden, PhD; James M.W. Brownjohn, PhD

Manuscript Region of Origin: 
Vibration Engineering Section - Office F111A

Department of Civil \& Structural Engineering

Sir Frederick Mappin Building

Mappin St.

Sheffield

S1 3JD

U.K.

Dear Sir/Madam,

Please accept the attached paper: 'ARMA Modelled Time Series Classification for Structural Health Monitoring of Civil Infrastructure', for review for publication in 'Mechanical Systems and Signal Processing'. The paper is focused on structural health monitoring, making use of time series response analysis to provide a statistical method of inferring structural change.

Kind Regards,

Dr Peter Carden 


\title{
ARMA Modelled Time Series Classification for Structural Health Monitoring of Civil Infrastructure
}

\author{
E. Peter Carden ${ }^{1}$ \\ James M.W. Brownjohn ${ }^{1}$ \\ ${ }^{1}$ Dept. of Civil and Structural Engineering, University of Sheffield, Sir Frederick Mappin \\ Building, Sheffield S1 3JD, UK
}

\begin{abstract}
Structural health monitoring (SHM) is the subject of a great deal of ongoing research leading to the capability that reliable remote monitoring of civil infrastructure would allow a shift from schedule-based to condition-based maintenance strategies. The first stage in such a system would be the indication of an extraordinary change in the structure's behaviour.

A statistical classification algorithm is presented here which is based on analysis of a structure's response in the time domain. The time series responses are fitted with Autoregressive Moving Average (ARMA) models and the ARMA coefficients are fed to the classifier. The classifier is capable of learning in an unsupervised manner and of forming new classes when the structural response exhibits change.

The approach is demonstrated with experimental data from the IASC-ASCE benchmark four story frame structure, the Z24 bridge and the Malaysia-Singapore Second Link bridge. The classifier is found to be capable of identifying structural change in all cases and of forming distinct classes corresponding to different structural states in most cases.
\end{abstract}


Keywords: Structural Health Monitoring; Time Series Modelling; ARMA models

\section{Introduction}

The last two decades have seen a great deal of research and publication in the field of SHM and there has been a proliferation of SHM paradigms put forward [1-3], with longterm monitoring systems implemented on bridges in Europe, the United States and Asia [4-6]. This has provided a great deal of practical experience and knowledge and helped SHM reach a greater level of maturity.

Despite this level of research, a robust method of indicating an adverse condition of a structure in service has yet to be demonstrated and widely implemented. This level of SHM is generally referred to as level 1 . The classification algorithm proposed in this paper is primarily aimed at this level but can also provide a heuristic guide to the condition of the structure depending on the level of prior knowledge.

Modeling of time histories is common in many disciplines and has been used to study various phenomena from sunspot activities to stock market indexes. The literature on the subject is extensive. A classic treatment is provided by Box et al. [7] while a more system analysis focused approach, and perhaps a more directly useful primer to the vibration engineer, is given by Pandit and $\mathrm{Wu}[8]$. 
It is possible to build linear stochastic models of structural time response histories using ARMA models if it is supposed that the structure is excited by a series of random shocks. An ARMA model of order $(p, q)$ is defined by equation (1).

$$
\phi(B) z_{t}=\theta(B) a_{t}
$$

$z_{t}$ is the time history response of the structure. $a_{t}$ is the series of Gaussian distributed random shocks exciting the structure. $\phi(B)$ is the autoregressive function of order $p$ defined in equation (2) and $\theta(B)$ is the moving average function of order $q$ defined in equation (3).

$$
\begin{aligned}
& \phi(B)=1-\phi_{1} B-\ldots-\phi_{p} B^{p} \\
& \theta(B)=1-\theta_{1} B-\ldots-\theta_{q} B^{q}
\end{aligned}
$$

$B$ is the backward shift operator: $B^{m} z_{t}=z_{t-m}$.

The autoregressive parameters are directly related to the system poles, $\lambda_{\mathrm{i}}, \mathrm{i}=1,2, \ldots \mathrm{n}$ through equation (4) [8]. For the case of dynamic structural response involving a number of vibration modes, $\mathrm{n}$ is twice the number of system modes excited and each mode is represented by a complex conjugated pair of poles.

$$
\phi_{l}=(-1)^{l+1} \sum_{\substack{i 1, i 2, \ldots i l=1 \\ i 1<i 2<\ldots<i l}}^{n} \lambda_{i 1} \lambda_{i 2} \ldots \lambda_{i l}
$$

Each pair of poles is related to the natural frequency, $\omega_{\mathrm{n}}$, and damping ratio, $\zeta$, of a mode through equation (5).

$$
\lambda, \lambda^{*}=e^{\Delta\left(-\zeta \omega_{n} \pm \omega_{n} \sqrt{\zeta^{2}-1}\right)}
$$


Where $\lambda^{*}$ is the complex conjugate of $\lambda . \Delta$ is the sampling time interval.

Thus, the natural frequency and damping ratio may be determined by examining only the autoregressive parameters. This has led to the moving-average parameters generally receiving less attention in the system identification literature despite their importance for modeling. For example, Fig. 1 shows the power spectral densities of single degree of freedom (SDOF) systems modeled as $\operatorname{ARMA}(2,1)$ systems. In this illustration the AR parameters are held constant while the MA parameter is varied over a range of 0.5 to 2.5 . The greater power in the signal with the MA parameter equal to 0.5 compared with 1.0 is not an error but rather results from the non-linear relationship between the MA parameter and the power in the mode. It should be noted that changing the MA parameter while maintaining the AR parameter values is equivalent to changing the stiffness, mass and damping properties of an SDOF system while maintaining the natural frequency and damping ratio values; the five spectral densities depicted cannot come from the same SDOF if the excitation is invariant. In a multiple degree of freedom system the amplitude response of each mode at a particular point is not equal and the MA parameters are required to model this phenomenon.

If a structure is excited by truly random Gaussian distributed noise and no noise is present in the measurement then the order of the autoregressive function $(p)$ will equal $n$ and the order of the moving average function $(q)$ will equal n-1 i.e. it may be modeled as an ARMA (n,n-1) process [8]. If noise is present in the measurement then it may be 
modeled as an extra moving average parameter i.e. as an ARMA(n,n) process [9]. In reality the excitation may not be truly Gaussian and may be imagined as Gaussian noise passing through a shaping filter before exciting the system. The poles of the shaping filter may then be modeled as extra ARMA parameters and thus $p, q>\mathrm{n}$. It is common when acquiring data to apply filtering to the signal, to prevent aliasing for example and such filters will introduce additional numerical poles into the measured response.

When dealing with real data measured on structures, it is therefore generally not possible to determine an exact model order a priori. A sufficient model order may be determined by examining the residuals $a_{t}$ on the input side of equation (1). If they do not have a random Gaussian distribution then the ARMA model has not captured the system dynamics, the excitation poles, the measurement noise, the filter characteristics or a combination of these.

The Akaike Information Criterion (AIC), the Bayesian Information Criterion (BIC) and Rissanen's Minimum Description Length (MDL) criterion may also help in determining suitable model orders [10]. Each of these criteria penalise larger model orders in different ways. Hence they may suggest different model orders and should be used with careful judgment. The examination of the residuals will be discussed in further detail when building models for the data from the ASCE Benchmark structure.

Changes in a structure's stiffness, mass or damping properties will change its modal properties. With the relationship between modal parameters and ARMA parameters 
expounded in equations (4) \& (5), it is clear that the ARMA parameters representing the dynamics of a structure may be used for health monitoring of that structure. Indeed AR and ARMA models have been fitted to historical response data from structures in undamaged states in order to create features for statistical process control, for example $[11,12]$. The residuals of the AR and ARMA models were used to form statistical process control charts and control limits were calculated by assuming the mean and variances of groups of the residuals to be normally distributed. This allowed the formation of confidence intervals for the undamaged structures' responses. The same stochastic models were then fitted to subsequent time history responses of the structures in damaged states. The resulting residuals were plotted on the control charts and those points lying outside of control limits were counted as outliers and used to indicate a change in the system and damage was successfully indicated. Mattson and Pandit [13] investigated the use of skewness and kurtosis as features for damage diagnosis but found them to be less reliable than the variance. Statistical process control charts do not allow the determination of the cause of structural response change. The classifier presented in this paper can provide such information if the time histories of the structure in such states have been previously measured.

The use of AR models rather than ARMA models is possible because the MA parameter series may be inverted to an AR parameter series. The equivalent series is theoretically of infinite length but in practice may be adequately estimated by a series of finite length. The actual length of the AR series needed to adequately describe the MA series is dependent on the magnitude of the MA parameters but is always longer the MA series. 
Superficially it would therefore seem rather misguided; however the advantage in using AR models without MA terms is that they may be estimated in a linear least squared manner whereas ARMA models must be estimated using non-linear least squared techniques. There is therefore a compromise between parsimony of model order and computational burden which the practitioner must balance. In this paper ARMA models are estimated using Ljung's System Identification Toolbox in Matlab [14].

Sohn and Farrar [15] modeled time series responses as AR-ARX processes which is equivalent to a linearization of an ARMA process. They set up a data bank of the ARARX parameters from previously modeled time series of an 8-DOF system in an undamaged state with different levels of force input. New data sets were then fitted with the closest AR-ARX set in the data bank. The standard deviation of the residuals from the new time series were compared with those from the old time series and a statistically significant difference indicated damage. Sohn et al. [16] employed a more sophisticated statistical test of the standard deviation of the residuals and showed successful results with a three-story laboratory frame structure.

The approach taken in these papers is very similar to that presented in this paper. The differences lie in the modeling strategy, (ARMA models are used in this paper), and in the method for distinguishing statistically significant differences between the residuals.

More recently, ARMA modeling was applied to the ASCE benchmark building [17] to detect and locate damage. The first AR coefficient was normalized by the square root of 
the sum of the squares of the first three AR coefficients to form a damage sensitive feature. Using the t-test with both analytical and experimental results, this feature was determined to be significantly different between signals from the structure in undamaged and damaged states. Furthermore, the location of damage was indicated from indices calculated from the relative changes in the first two AR coefficients. When compared to baseline values for the structure in a healthy state, the indices showed greater changes in value for time histories recorded from sensors closest to the location of damage rather than those further away. The method was successful, although the methodology employed to fit the ARMA models was rather unorthodox. Finite moving average windows were applied to the time histories to remove cyclical trends seen in the autocorrelation plots. If seasonal trends had been present, such as diurnal temperature changes, then this strategy would be fully justified. The damped sine wave structures present in the autocorrelation plots are to be fully expected in over-damped systems with complex conjugate poles and it is unnecessary to remove them $[7,8]$. Due to the windowing, the resulting ARMA coefficients are difficult to interpret directly for physical meaning and thus the almost arbitrary sounding normalization of the AR coefficients would seem to have been necessary. Finite moving average windows are not applied to the time histories in this paper and a sound physically based modeling strategy is employed.

The basis of a classification algorithm using a statistical test on the residuals of the model is presented in the following section. The algorithm is then demonstrated on experimental 
data from the ASCE benchmark 4 story structure[18], the Z24 bridge[19] and the Malaysia-Singapore Second Link bridge[20].

\section{Classification Algorithm}

If a structural system changes in some way then clearly so will its ARMA parameters. A classification of the ARMA parameters is therefore a logical approach to monitoring the health of a structure. A statistical test is needed to determine such changes as being significant or not as ARMA parameters estimated from measured time series have natural variability. The variability will be lower for longer time series and the estimated coefficients will converge on the true process values as the length of the time series tends to infinity. For instance the analytically derived variance for the MA coefficient, $\theta$, of an MA(1) process is $\left(1-\theta^{2}\right) / \mathrm{n}$, where $\mathrm{n}$ is the number of samples in the time series [7]. To the authors' knowledge it is not possible to derive rigorous expressions for analytical variance of general ARMA models. However, Box et al. [7] Section 7.1.7, demonstrated that approximate $1-\varepsilon$ confidence regions of the ARMA parameters are bounded by a contour on the surface of the sum of the squares of the residuals given by equation (6):

$$
S(\boldsymbol{\beta})<S(\hat{\boldsymbol{\beta}})\left[1+\frac{\chi_{\varepsilon}^{2}(k)}{n}\right]
$$

$S($.$) is the sum of the squares of the residuals function, \boldsymbol{\beta}$ are the ARMA parameters estimated from a time series of length $n$ and $\hat{\boldsymbol{\beta}}$ are the previously known ARMA parameters from a particular process. Generally, only an estimate of $\hat{\boldsymbol{\beta}}$ is known. $\chi_{\varepsilon}^{2}(k)$ 
is the significance point exceeded by a proportion $\varepsilon$ of the $\chi^{2}$ distribution, having $k$ degrees of freedom. $\mathrm{k}$ is equal to the order of the ARMA model. $\varepsilon$ is chosen as $1 \%$ in the systems examined later.

Equation (6) allows the comparison of all the ARMA parameters simultaneously. If it is postulated that the time series is generated by a process with true ARMA parameters $\hat{\boldsymbol{\beta}}$, which means it is a structure in a healthy state in our context, then equation (6) tests whether the parameters $\boldsymbol{\beta}$ are significantly statistically similar or different to $\hat{\boldsymbol{\beta}}$, in other words healthy or not healthy.

The ARMA parameters consist not only of the structure's dynamic characteristics but also characteristics of excitation, noise and filtering as already stated. If these other characteristics are inconsistent, say the variable excitation of a bridge by traffic, then new poles of low energy may appear or disappear in the signal. Because of their low energy content, the inequality in equation (6) may still hold, however the direct comparison of ARMA parameters is not always possible. For this reason it may be sometimes possible to average $\boldsymbol{\beta}$ from several time series to get an improved estimate of $\hat{\boldsymbol{\beta}}$, but not in other cases. It is desirable to get a better estimate $\hat{\boldsymbol{\beta}}$ rather than hold many sets of $\boldsymbol{\beta}$ separately as it would reduce the error of falsely assessing a time series as being from the same process when it is in fact from a different process. If a class corresponding to the structure in a healthy state were set it up it would therefore contain several sets of $\hat{\boldsymbol{\beta}}$ 
values each of which was estimated from several time series. This explains Step 5(b) in the classification algorithm described below.

Equation (6) will be used as the basis for a classification algorithm for SHM assuming that ARMA models can be fitted to the responses of the structure being measured. If a number of time series from the structure are available, the classifier may be run in an unsupervised learning mode according to the following steps.

Step 1: Estimate $\boldsymbol{\beta}$ for the first time series and use these to form Class 1 where $\hat{\boldsymbol{\beta}}_{11}$ is equal to $\boldsymbol{\beta}$.

Step 2: Feed another time series to the classifier, estimate $\boldsymbol{\beta}$ and $S(\boldsymbol{\beta})$ for the new time series.

Step 3: Estimate $S\left(\hat{\boldsymbol{\beta}}_{c a}\right), c=1,2, \ldots \mathrm{m} ; a=1,2, \ldots \mathrm{n}$. Where $\mathrm{m}$ is the number of classes previously formed by the classifier and $\mathrm{n}$ is the number of sets of parameters held in class $c$.

Step 4: Use equation (6) with $S(\boldsymbol{\beta})$ and $S\left(\hat{\boldsymbol{\beta}}_{c a}\right)$, for all $c$ and $a$, and see if the inequality holds.

Step 5: If the inequality holds for any parameter set $a$ in class $c$ then:

(a) Classify the signal in that class;

(b) If the difference between each element of $\boldsymbol{\beta}$ and $\hat{\boldsymbol{\beta}}_{c a}$ is less than $\mathrm{z} \%$ then update $\hat{\boldsymbol{\beta}}_{c a}$ by averaging in $\boldsymbol{\beta}$; if it greater than z\% then add $\hat{\boldsymbol{\beta}}_{c(n+1)}$ equal to $\boldsymbol{\beta}$ to class c. 
If the inequality doesn't hold, form a new class with $\hat{\boldsymbol{\beta}}_{(m+1) 1}$ equal to $\boldsymbol{\beta}$.

Step 6: Repeat steps 2 to 5 for the number of series required.

$\mathrm{z} \%$ is chosen as $20 \%$ in the classification examples in this paper. The classifier was found to work with many values of $z \%$ from $5 \%$ to $50 \%$ and its chosen value was not found to be critical to the performance of the classifier. No attempt to ascertain an optimal value has been made and it is likely that a more suitable criterion could be developed.

Nevertheless, this criterion works sufficiently well in the applications presented in this paper.

If the structural response changes due to damage or structural deterioration such as bearing wear, extraordinary loading, environmental variables etc, then the classifier should form a new class. For the purposes of SHM it would be desirable to separate the effects of environmental variables such as temperature from other causes of change in structural response. To achieve this, a database containing classes formed from the structure in a healthy, normally loaded state over a period of varying environmental conditions could be stored. New time series responses from the structure could then be compared to this database. The formation of the database would involve an extended period of initial monitoring. This task is beyond the scope of this present paper and is the subject of current research by the authors.

In operation, the first estimate, $\hat{\boldsymbol{\beta}}_{c p}$, of each class may be considerably different to the true $\hat{\boldsymbol{\beta}}_{c}$ of that class. As a result, several classes may be initially formed which all 
correspond to the same state of the structure. However, after more series are passed to the classifier the estimates of $\hat{\boldsymbol{\beta}}_{c}$ will converge towards the true value of $\hat{\boldsymbol{\beta}}_{c}$ and each of these classes will therefore also converge towards each other. The examples presented in this paper all exhibit this phenomenon. In particular, the process is explicitly presented in the Malaysia-Singapore Second Link case study. Two classes were initially formed for the structure in a 'healthy' state. The values of the single AR and single MA parameters in the two classes were quite different on formation (if they were not, separate classes would not be formed). After the classifier received 15 series from the structure in a 'healthy' state, the single AR and single MA parameters in the two classes converged towards the same values. It is not explicitly shown in the other two case studies because of the number parameters involved (the ARMA model orders are much larger) and the limited space available.

The classification approach is demonstrated with experimental dynamic data from the ASCE Benchmark structure and the Z24 Bridge in various states of damage. The testing of these structures was undertaken over a short period of time and thus the changes in the environmental variables were limited. The classification approach is also demonstrated with static data from the Malaysia-Singapore Second Link Bridge recorded over a period of seven years. Both creep and diurnal trends in the data are incorporated in the time series modeling.

\section{Damage Classification of the IASC-ASCE Benchmark Structure [18]}


The IASC-ASCE benchmark structure is a four-story, two-bay by two-bay steel-frame scale model structure built in the Earthquake Engineering Research Laboratory at the Univ. of British Columbia, Canada. The structure is $2.5 \times 2.5 \times 3.6 \mathrm{~m}$ in size (see Fig. 2). A full description of the experiment is given in [18]. A total of 9 test configurations were investigated and are listed in Table 1, where the structure may be considered to be in an undamaged 'healthy' state in configuration 1. The structure was excited in several ways but this paper uses only those responses obtained from an electrodynamic shaker placed roughly at the center of one of the four bays on the top level of the structure..

Data from this structure have been examined by other researchers. Ching et al. [21] reported that due to shaker-structure interaction, the shaker input force data were contaminated by the structural response. Consequently the extracted mode shapes were irregular, leading to difficulties in damage detection unless the shaker-structure interaction can be accounted for and recognised in the model. When fitting ARMA models to the time response this interaction is inherently modeled and does not present a problem.

The structure was instrumented with 15 accelerometers though only the results from Channel 14 , located on the $4^{\text {th }}$ floor, are presented in this paper. The data were acquired with a sampling frequency of $200 \mathrm{~Hz}$ and a large number of modes of the structure were excited. If an ARMA filter is fitted to these data directly then a large order is required. To avoid this, the time series were decimated by four with a low pass Chebyshev filter. This order of decimation was chosen to remove modes higher then approximately $25 \mathrm{~Hz}$ from 
the signal. Of course shifts in these modes would be beneficial in detecting changes in the structure but it was found that the information below $25 \mathrm{~Hz}$ is sufficient for correct classification here and the reduction in the model order is beneficial. Without this decimation a model order of between 30 and 40 would be required.

The algorithm described here will essentially rely on shifts of the modal parameters of the first three modes. The decimation resulted in a collection of signals with 6000 samples. These were then broken into three segments of 2000 each. Thus, there were 27 time series for the classifier to classify.

As discussed earlier, a key decision in time series modeling is choosing the model order. The autocorrelation and partial autocorrelation functions are often used to help choose model order [7] but for large ARMA orders these are difficult, if not impossible, to interpret. Fig. 3 shows the autocorrelation plots for the structure in configuration 1 using all 6000 samples and, for comparison, just the first 2000 samples. The damped sine wave structures are present at the start of the plots. In theory they should die out, but it is clear from the Fig. 3 that while they do decay they do not tend to zero. A possible cause of this effect is given in [22] as being due to the shortness of the series; it represents a numerical chimera. The apparent structure at high lags is due to the series not being infinite and should be ignored. There are clear differences in the autocorrelation plots when comparing Fig. 3(a) with Fig. 3(b) due to the different sample sizes, further illustrating the point. The partial auto correlations are equally unhelpful in choosing model order and are not presented here. 
Fig. 4 shows the auto power spectral density from the first 2000 samples of the time series of configuration 1 . There are three clear large poles in spectrum before the energy dies out towards the filter cut off frequency of $25 \mathrm{~Hz}$. We would therefore expect a model order of at least ARMA $(6,6)$. Using the AIC also suggests a model order of $\operatorname{ARMA}(6,6)$, however when this model order is fitted to the data, the residuals do not appear to be Gaussian distributed. Fig. 5 shows the correlation function of the residuals with $99 \%$ confidence limits for a) $\operatorname{ARMA}(10,10)$ and b) $\operatorname{ARMA}(6,6)$ models . The $\operatorname{ARMA}(10,10)$ model seems far closer to providing Gaussian residuals than does the ARMA $(6,6)$ model with the additional poles predominantly due to the Chebyshev filter. In the following analysis $\operatorname{ARMA}(10,10)$ models were fitted to the data, though in fact the $\operatorname{ARMA}(6,6)$ models performed equally well. The mean value for each series was removed before each ARMA model was fitted.

The 27 time series segments of available data were fed to the unsupervised classifier and the results are presented in Table 2 . The classes are formed automatically in an unsupervised manner and therefore their interpretation is up to the user. Ideally just one class would be formed for each of the nine configurations. The estimates of $\hat{\boldsymbol{\beta}}$ generated for each class are unlikely to be very accurate for two reasons:

(a) only three time series were available for each configuration;

(b) each series was only 2000 in length.

This inaccurate estimation led to the formation of more than one class per configuration. Given a larger number of series, the $\hat{\boldsymbol{\beta}}$ estimates for different classes corresponding to the 
same configuration are likely to converge toward each other. Thus in the long term the number of classes corresponding to a particular configuration would converge to one.

No damaged configuration was classified as undamaged and all series from configuration 1 (undamaged) were classified together. The algorithm can therefore be judged as being successful though testing with larger quantities of data is necessary to better judge its performance.

\section{Damage Classification of the Z24 Bridge [19]}

The Swiss Z24 bridge was constructed from prestressed concrete, with spans of 14-3014m. Prior to its demolition in 1998 it was the subject of extensive testing including 16 progressive damage tests (PDTs). The testing is described in detail in [19] and the PDTs are listed in Table 3. The installation of testing apparatus involved serious changes in the structure. Due to this, three of the PDTs in Table 3 are described as reference states to which other PDTs are compared. The structure is considered to be 'healthy' in the reference states and damaged in the other PDTs.

As part of the progressive damage, jacks were installed on the pier at the Koppigen end of the bridge to facilitate reversible settlement of the pier. After PDTs 3 to 6, which involved lowering of the pier and produced some cracking, the pier was returned to its original position. The foundation was then rotated in PDT 7. The foundation was returned to its original position before a reference measurement was taken, denoted as PDT 8. PDTs 9 to 16 involved irreversible, cumulative damage. It is therefore natural to break 
the data set into two parts to test the classifier, namely: PDTs 2 to 7 and PDTs 8 to 16 . PDT 1 is left out as the installation of the jack in the pier and associated safety work caused a large change in the dynamic characteristics of the bridge. The classifier distinctly and clearly separates PDT 1 from PDT 2 so the comparison of PDT 1 with other PDTs is without interest.

For each PDT, the bridge was tested with both ambient and forced excitation. A set of roving accelerometers along with three reference locations were used in nine test setups for each PDT. Data were sampled at $100 \mathrm{~Hz}$ and a total of 65568 samples were measured for each setup. In this paper the data from reference accelerometer 1 located near the Koppigen pier recorded during forced excitation are used and 72 series of length 8000 each were extracted from this data set for each PDT. The auto-power spectra showed little structure beyond $25 \mathrm{~Hz}$ (i.e. no modes of the structure beyond this frequency were significantly excited) and so each series was decimated by two without filtering in order to avoid introduction of additional poles. The aliasing of noise energy was considered the lesser of two evils. Examination of the residuals with progressively increasing model order led to a choice of fitting the series with an $\operatorname{ARMA}(30,30)$ model. The average of each series was removed before each ARMA model was fitted.

\subsection{Performance of the classifier on PDTs 2 to 7}

PDTs 3 to 6 involved progressive settlement of the pier. The classifier classed 23 of the 72 time series of PDT 3 as the same as PDT 2 (the reference undamaged state). None of the time series from PDTs 4 to 6 were classed with PDT 2. This is encouraging as the 
classifier learnt in an unsupervised manner and as such, gradual damage will tend to cause the class to shift gradually and therefore fail in flagging damage. However the classifier was successful here as the step changes in settlement were large enough.

None of the series from PDT 6 were cross-classified with any other PDT. Two of the series from PDT 5 were classified with PDT 4. However, 52 of the series from PDT 4 were classified as the same as PDT 3. The classifier was therefore successful in distinguishing the larger magnitudes of settlement as distinct and separate but had more difficulty with lower magnitudes of settlement, which is unsurprising.

After the pier was returned to its original position, the foundation was rotated in PDT 7. The classifier classed all 72 series separately from PDT 2 (the reference undamaged state) and PDTs 3 to 6 . It was therefore completely successful in identifying this damage event.

\subsection{Performance of the classifier on PDTs 8 to 16}

The classifier formed 2 classes for PDT 8, the reference case. Of the 72 series fed to the classifier, 43 were double classified in class 1 and 2 . The classes converged towards each

other i.e. the $\hat{\boldsymbol{\beta}}_{c}$ values for classes 1 and 2 became very similar. As such, the two classes are inseparable at the end of classification and the signals classed in both classes should be considered to be classed together. An automatic merging of classes has not been programmed into the classifier algorithm though such a mechanism would be an improvement. 
PDTs 9 and 10 involved simulated spalling of concrete. All 72 series from each case were classified in classes 1 and 2 i.e. the classifier was unable to identify the occurrence of this type of damage. Kramer et al. [19] identified very small changes in the modal parameters of the first six modes in these PDTs and this is consistent with the statistically nonsignificant change in the ARMA model found here.

No series from PDTs 11 to 16 were classed in classes 1 or 2 . The classifier can therefore be considered completely successful in identifying the presence of damage in these cases, that is failure of concrete hinges, anchor heads and post tensioning wires.

4.3. Performance of the classifier in separating the damage cases

PDT 11, the simulated landslide, was distinctly and clearly classed separately from all the other PDTs. 23 of the 72 series from PDT 13 were classed together with PDT 12 despite these being different types of damage. Only one series from PDT 14 was classed with PDTs 13 and 12 and so severity of damage and not type of damage would seem to be more distinctive in this case. Interestingly, 68 series from PDT 15 and all 72 series from PDT 16 were classified as being the same as PDT 12. This is despite these PDTs being (the) more severe. However the shifts in the first six modes identified in [19] in PDTs 15 and 16 are very similar to those in PDT 5.

It is clear that the ARMA model based classifier as used in this case, and with the IASCASCE Benchmark structure, is relying on changes in the modal parameters of the 
structure due to damage. When these changes have not been significant, as in the case of the spalling of concrete on the Z24 bridge, the classifier has failed. Additionally, when the modal parameter shifts caused by different types of damage are similar, as is the case with PDTs 15 and 16 compared with PDT 5 in the Z24 case study, the classifier fails to distinguish between them. In contrast the next case study makes use of static data and therefore does not rely on these shifts in modal parameters.

\section{Monitoring of the Singapore-Malaysia Second Link Bridge [20]}

The Singapore-Malaysia Second Link Bridge, also referred to as the Tuas Link serves as a vehicular crossing between the Island of Singapore and Malaysian Peninsula, and is located in the Western side of the island. Fig. 7 shows the bridge under construction. The bridge was completed in 1997 and opened to traffic in the same year. Only the basic outline of the structure and the monitoring program undertaken are provided here and interested readers may find more details in [20]. The bridge is about $1.9 \mathrm{~km}$ long and comprises 27 spans; the Singapore side is about $170 \mathrm{~m}$ long and the main span of this section is $92 \mathrm{~m}$ long. The bridge was cast in-situ using the balanced cantilever method, to enable the navigation channel to be kept free throughout the construction, and posttensioned. The cross-section of the post-tensioned, continuous box girder varies in depth from $2.6 \mathrm{~m}$ to $6.5 \mathrm{~m}$ along the bridge length.

A suite of instruments was installed in the bridge in order to monitor its short-term and long-term performance under environmental and traffic loads in terms of stress (pressure cell) and strain (vibrating wire gauge) signals recorded at hourly intervals. Hence in contrast to the two previous examples, the classifier is applied to static data. The readings 
from the pressure cell located in Segment 31 over the course of approximately seven years are presented in Figure 5(a). Figure 5(b) shows a zoom in the recording data over a shorter period. There are several gaps in the data record due to the monitoring being stopped for various readings. At approximately 21000 hours the tensioning of the final segment (stitching) took place and a significant rise in the pressure cell reading is evident. After this rise there is a distinct downward trend in the pressure readings which is believed to be due to creep. Additionally the data show a diurnal trend. These trends necessitate an extension of the modeling technique used previously. Omenzetter et al. [23] used an ARIMA model on the same data set and this approach is also used here. A minimum amount of detail of the modeling is presented here and the interested reader is referred to [23] for more thorough discussion.

The linear trend due to creep may be eliminated by differencing the data using the operator $\nabla_{s} z_{t}=z_{t}-z_{t-s}$ with $s=1$ [7]. The diurnal trends may be accounted for by differencing with $s=24$ and by augmenting equation (1) with seasonal autoregressive and moving-average functions denoted by superscript $s$ as in equation (7).

$$
\phi^{s}(B) \phi(B) z_{t}=\theta^{s}(B) \theta(B) a_{t}
$$

Seasonal ARIMA model orders are commonly denoted by $(p, d, q) \mathrm{x}(p, d, q)_{\mathrm{s}}$ where $p$ is the order of the autoregressive function, $d$ the order of differencing, $q$ the order of the moving-average function and subscript $\mathrm{s}$ is short for seasonal. As the data are static we cannot define a minimum order based on the number of modes excited. However, static data will generally have far lower order than dynamic data and so the examination of the autocorrelation and partial autocorrelation functions provide more insight. Examination 
of the residuals and of the change in AIC with model order are also useful. Omenzetter et al. [23] found a model of order $(1,1,0) \times(0,1,1)$ fitted the data well and this order is also used here. It may be noted that the time variance of the ARIMA coefficients was examined using a Kalman filter in [23]. This is radically different from the approach used here.

Seventeen blocks of data of length 1000 were selected from the recorded pressure cell and are denoted by vertical lines in Fig. 8(a). Thus each data block represents approximately six weeks of recordings. Fig. 8(b) shows a zoom in on a typical section of recorded data. One of the selected data blocks was generated before the final stitching of the bridge, while the other 16 are spread over the remaining seven years of monitoring. As the bridge was subject to an unknown and varying ambient excitation, and not a constant level of forced excitation as in the previous two examples, the data blocks were normalized by subtracting their average and dividing by their standard deviation.

The classifier formed two classes for the data blocks recorded after the final stitching of the bridge and a third class for the data block recorded prior to the stitching. Only one vector of coefficients was held in each class i.e. during Step 5(b) of the classification algorithm the maximum differences between the elements of $\boldsymbol{\beta}$ and $\hat{\boldsymbol{\beta}}_{c p}$ were always less than $50 \%$. Fifteen of the 16 post-stitching data blocks are classed in class 1, 10 are classed in class 2 and 9 are classed in both classes 1 and 2. On initial formation, the ARMA coefficients of class 1 were $A R=-0.7497$ and $\mathrm{MA}_{\mathrm{s}}=-0.8674$ while those of Class 2 were $\mathrm{AR}=-0.7000$ and $\mathrm{MA}_{\mathrm{s}}=-0.9511$. The convergence of classes 1 and 2 towards each other at the end of the unsupervised learning period is clearly seen in Table 4. Class 3 , 
which is formed from just 1 data block prior to stitching of the bridge, is very clearly different to classes 1 and 2 even without the use of a statistical tool to distinguish them.

The classification algorithm has undoubtedly been successful in grouping together pressure cell recording from the bridge in a 'healthy' state while distinguishing a data block from the bridge prior to its final completion. However, the final stitching of the bridge together represents a massive change in structural behaviour and the sensitivity of the ARMA coefficients of such static data to more minor changes remains open to further investigation.

\section{Discussion}

Both the IASC-ASCE Benchmark structure and the Z24 bridge data were recorded during forced excitation. This resulted in an almost constant level of input energy across all the test configurations and the classification algorithm was successful in general. The advantage of this approach over examining the changes in the natural frequency, damping and mode shape of each mode is that it conveniently takes all of these features for all modes in the frequency spectrum examined and compresses them into a single statistical test in the form of equation (6). The ARMA model also contains information on the excitation, digital filter characteristics and noise in the measurement. It is conceivable to consider the digital filter characteristics and the noise level to be fairly constant and therefore not to cause significant error in classification. The inherent modeling of the shaker-structure interaction in the case of the IASC-ASCE Benchmark structure was helpful. Additionally it is foreseeable that the flagging of an extraordinary load on a bridge would be beneficial to a bridge manager. 
However, these benefits do not come without some disadvantage. It would be far more cost effective to be able to rely on ambient excitation of structures rather than forced excitation for continuous, long term, remote health monitoring. The ambient excitation will undoubtedly vary in time, not only in amplitude but also in the frequency spectrum excited. A typical example is day time traffic compared to night time traffic on a bridge. The change in amplitude of excitation will result in a change in the MA parameters as is clearly seen for the SDOF systems in Fig. 1. It could be imagined that a large database of time series from the structure in a healthy state under typical varying excitation conditions could be formed to avoid false alarms. This database would also account for varying environmental conditions. However, it could be argued that the ARMA classification is relying on shifts in the natural frequencies and damping estimates alone in this case and directly extracting these characteristics from the data would be a more computationally efficient approach. Such an approach, accounting for the effects of environmental variables such as temperature, was successfully presented for the Z24 bridge in [24].

It would therefore seem that the use of static data, as seen in the Malaysia-Singapore Second Link Bridge example, may be the more fruitful approach for ARMA model based health monitoring in the future. The model was able to account for the long term trend of creep and the diurnal trends in the data. Insensitivity to environmental variables in the example may have been enhanced by taking data in blocks recorded over 6 week periods. However, this was necessary to provide a sufficient number of data points (1000) to form an ARIMA model with reasonable variance of its coefficients. The sensitivity of such 
static measurements to more minor structural changes than that presented is unproven and is the subject of current research by the authors.

\section{Conclusion}

A sound physical basis for forming ARMA models of structural response data has been presented. The use of these ARMA models in distinguishing a structure in a healthy state and various states of ill-health has been demonstrated. The health monitoring algorithm is based on a solid statistical test of the sum of the square of the residuals. Two cases studies using recorded dynamic data were presented. The algorithm was generally successful in identifying the occurrence of damage and in separating the different damage events from each other. However, the data were recorded from forced excitation tests and the approach may not be the most suitable SHM paradigm for structures with only ambient dynamic excitation. The approach also proved successful when used on static data recorded from a bridge over a 7 year period. The algorithm successfully distinguished the response of the bridge under operating conditions from the response of the bridge prior to completion. The model incorporated a long term linear trend due to creep and a diurnal trend and the classifier did not trigger false alarms due to these trends. The sensitivity of ARMA models of static response data to typical infrastructural damage is unproven and is the subject of current investigation by the authors.

\section{References}

1. S.W. Doebling, C.R. Farrar, M.B. Prime, D.W. Shevitz, Damage Identification and Health Monitoring of Structural and Mechanical Systems from Changes in their 
Vibration Characteristics: A Literature Review, Los Alamos National Laboratory Technical Repor, LA-13070-MS, 1996.

2. H. Sohn, C.R. Farrar, F.M. Hemez, D.D. Shunk, D.W. Stinemates, B.R. Nadler, A Review of Structural Health Monitoring Literature: 1996-2001, Los Alamos National Laboratory Report, LA-13976-MS, 2003.

3. E.P. Carden, P. Fanning, Vibration Based Condition Monitoring: A Review, Structural Health Monitoring 3(4) (2004) 355-377.

4. J.M.W. Brownjohn, P. Moyo, P. Omenzetter, S. Chakraborty, Lessons from Monitoring the Performance of Highway Bridges, Structural Control and Health Monitoring 12 (2005) 227-244.

5. J.M. Ko, Y.Q.Ni, Technology Developments in Structural Health Monitoring of Large-scale Bridges, Engineering Structures 27 (2005) 1715-1725.

6. Hovhanessian G, Health monitoring of cable-stayed structures. Experience and implementation, in: Proceedings of IMACXXIV, St. Louis USA, January, 2006.

7. G.E.P. Box, G.M. Jenkins, G.C. Reinsel, Time Series Analysis: Forecasting and Control, Prentice-Hall, New Jersey, USA, 1994.

8. S.M. Pandit, S.M. Wu, Time Series and System Analysis with Applications, John Wiley \& Sons, New York, USA, 1983.

9. P. Andersen, Identification of civil engineering structures using vector ARMA models, PhD Thesis, Department of Building Technology and Structural Engineering, Aalborg University, Aalborg, Denmark, May, 1997.

10. L. Ljung, System Identification: Theory for the User, $2^{\text {nd }}$ ed., Prentice-Hall, New Jersey, USA, 1999. 
11. K. Worden, G. Manson, N.R.J. Fieller, Damage Detection Using Outlier Analysis, Journal of Sound and Vibration 229(3) (2000) 647-667.

12. M.L. Fugate, H. Sohn, C.R. Farrar, Unsupervised Learning Methods for Vibrationbased Damage Detection, in: Proceedings of the $18^{\text {th }}$ International Modal Analysis Conference, San Antonio, Texas, USA, February, 2000.

13. S.G. Mattson, S.M. Pandit, Statistical Moments of Autoregressive Model Residuals for Damage Localization, Mechanical Systems and Signal Processing, 20(3) (2006) $627-645$

14. Ljung, L., System identification Toolbox, The Mathworks, Inc., 2000.

15. H. Sohn, C.R Farrar, Damage diagnosis using time series analysis of vibration signals, Smart Materials and Structures 10 (2001) 446-451

16. H. Sohn, D.W. Allen, K. Worden, C.R. Farrar, Statistical Damage Classification Using Sequential Probability Ratio Tests, Structural Health Monitoring, 2(1) (2003) $57-74$

17. K.K. Nair, A.S. Kiremidjian, K.H. Law, Time Series-based Damage Detection and Localization Algorithm with Application to the ASCE Benchmark Structure, Journal of Sound and Vibration, In Press, Available online: 12 September 2005.

18. S.J. Dyke, D. Bernal, J.L. Beck, C. Ventura, Experimental phase of the structural health monitoring benchmark problem, in: Proceedings of the 16th Engineering Mechanics Conference, ASCE, Reston, Va, 2003.

19. C. Kramer, C.A.M. de Smet, G. de Roeck, Z24 Bridge Damage Detection Tests, in: Proceedings of the $17^{\text {th }}$ International Modal Analysis Conference (IMAC), Kissimee, Florida, pp. 1023-1029, 1999 
20. P. Moyo, J. M. W. Brownjohn, Detection of Anomalous Structural Behavior Using Wavelet Analysis, Mechanical Systems and Signal Processing, 16(2/3) (2002) 429445.

21. J. Ching, J.L. Beck, Bayesian Analysis of the Phase II IASC-ASCE Structural Health Monitoring Experimental Benchmark Data, Journal of Engineering Mechanics, 130(10), October, 2004

22. M.G. Kendall, On Autoregressive Time Series, Biometrika, 33(2) (1944) 105-122

23. P. Omenzetter, J.M.W. Brownjohn, Application of time series analysis for bridge monitoring, Smart Materials Structures 15 (2006) 129-138

24. B. Peeters, G. De Roeck, One-year monitoring of the Z24-Bridge: environmental effects versus damage events, Earthquake Engineering And Structural Dynamics, 30 (2001) 149-171 


\section{List of Figures}

Figure 1 Auto-power spectral densities of SDOF systems modelled with varying values of the MA parameter while maintaining the AR parameter values.

Figure 2 IASC-ASCE Four-Story Experimental Benchmark Structure (Courtesy of Prof. Shirley Dyke, Washington University St. Louis)

Figure 3 Autocorrelation of the decimated time series from channel 14 of the ASCE structure in an undamaged state (a) 6000 samples (b) 2000 samples

Figure 4. Auto-power spectral density of channel 14 of the ASCE structure in an undamaged state

Figure 5 Correlation function of residuals from fitting ARMA models to the decimated time series of channel 14 of the ASCE structure (a) Order $(10,10)(b) \operatorname{Order}(6,6)$

Figure 6 The Z24 Bridge (Courtesy of Prof. Guido De Roeck, Katholieke Universiteit Leuven)

Figure 7 The Malaysia-Singapore Second Link under construction

Figure 8 (a) Pressure Cell readings in segment 31 of the Malaysia-Singapore Second Link (b) Zoom in of a typical section of recorded data 


\section{List of Tables}

Table 1 Test Configurations of the ASCE Benchmark Structure

Table 2 Classification of 27 time series from the ASCE structure in 9 structural

\section{Configurations}

Table 3 Progressive Damage Tests (PDTs) of the Z24 Bridge

Table 4 The ARMA coefficients for each class formed from the Malaysia-Singapore

Second Link data 
Figure 1
Click here to download high resolution image

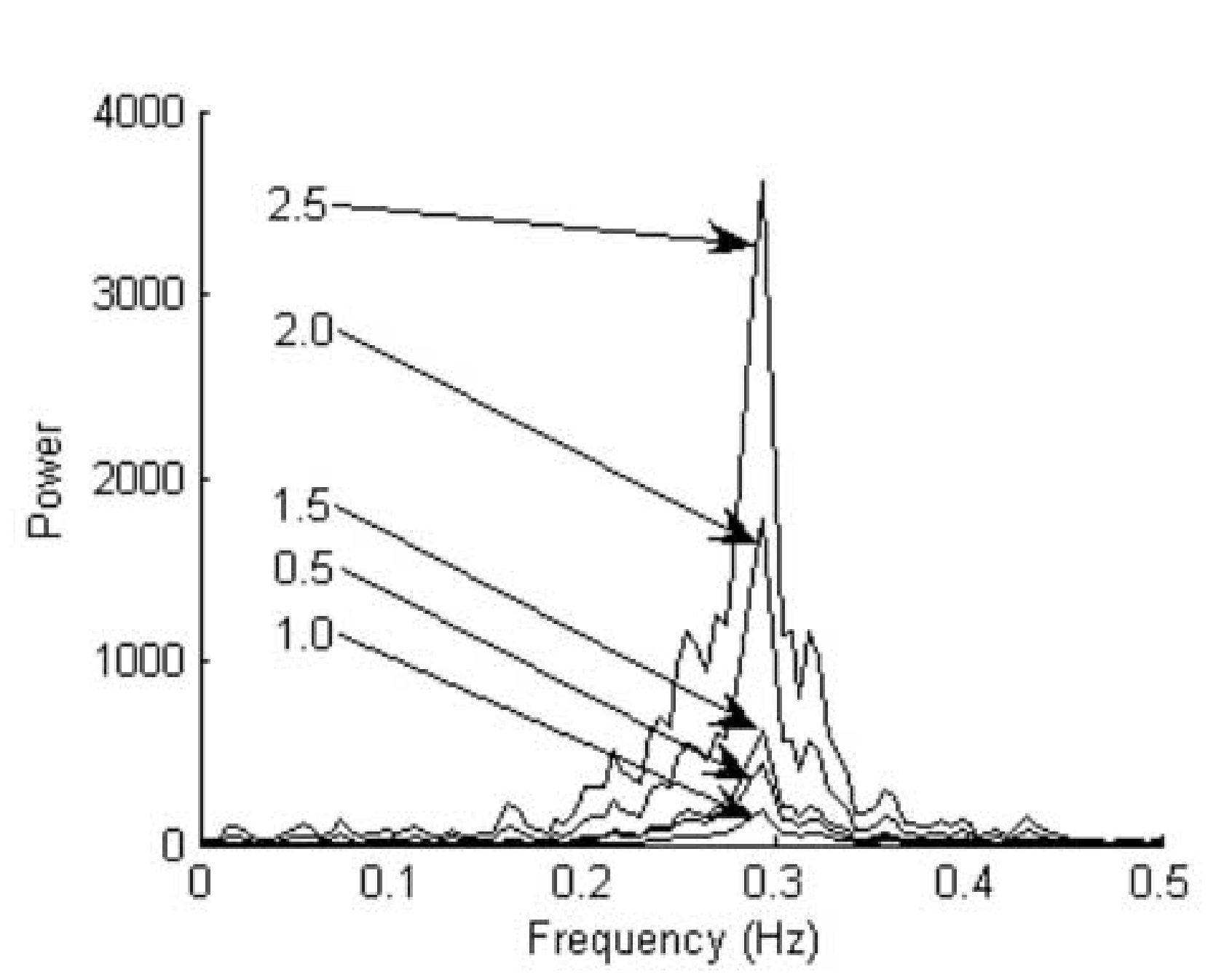

Click here to download high resolution image

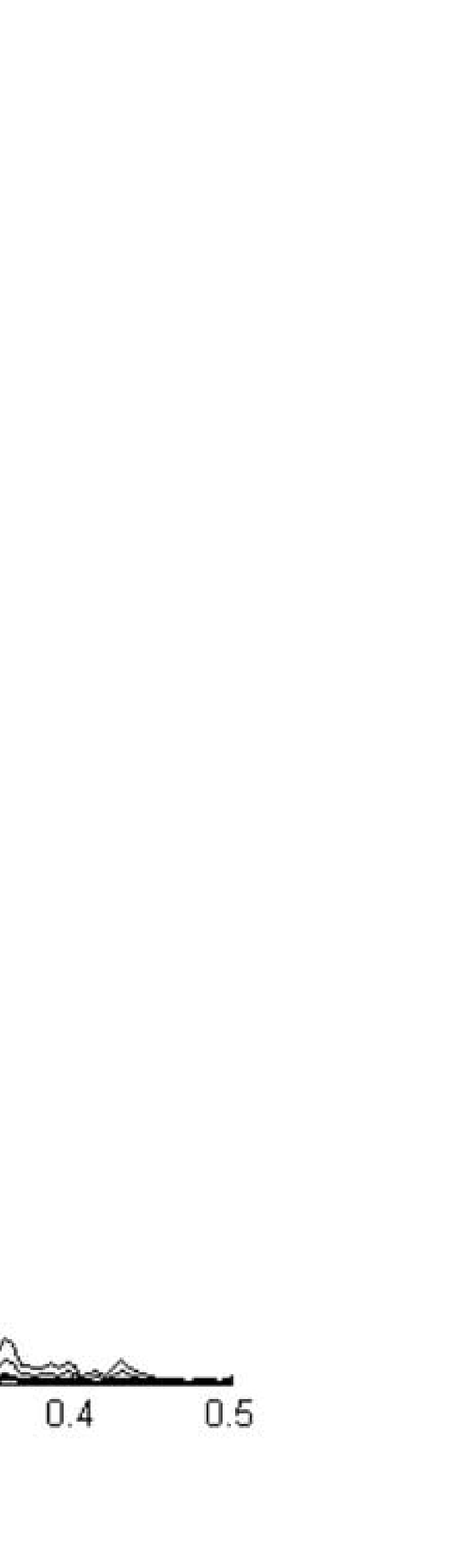




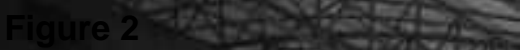
(N) -
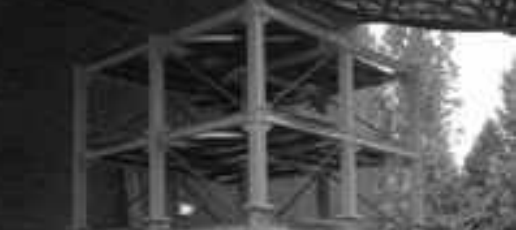

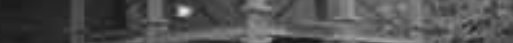

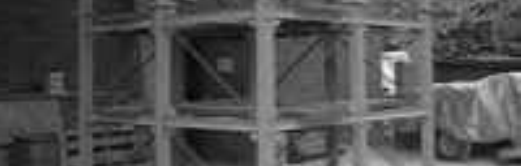

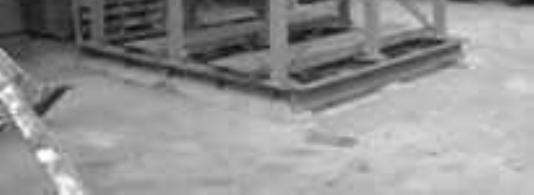


Figure $3 a$

Click here to download high resolution image

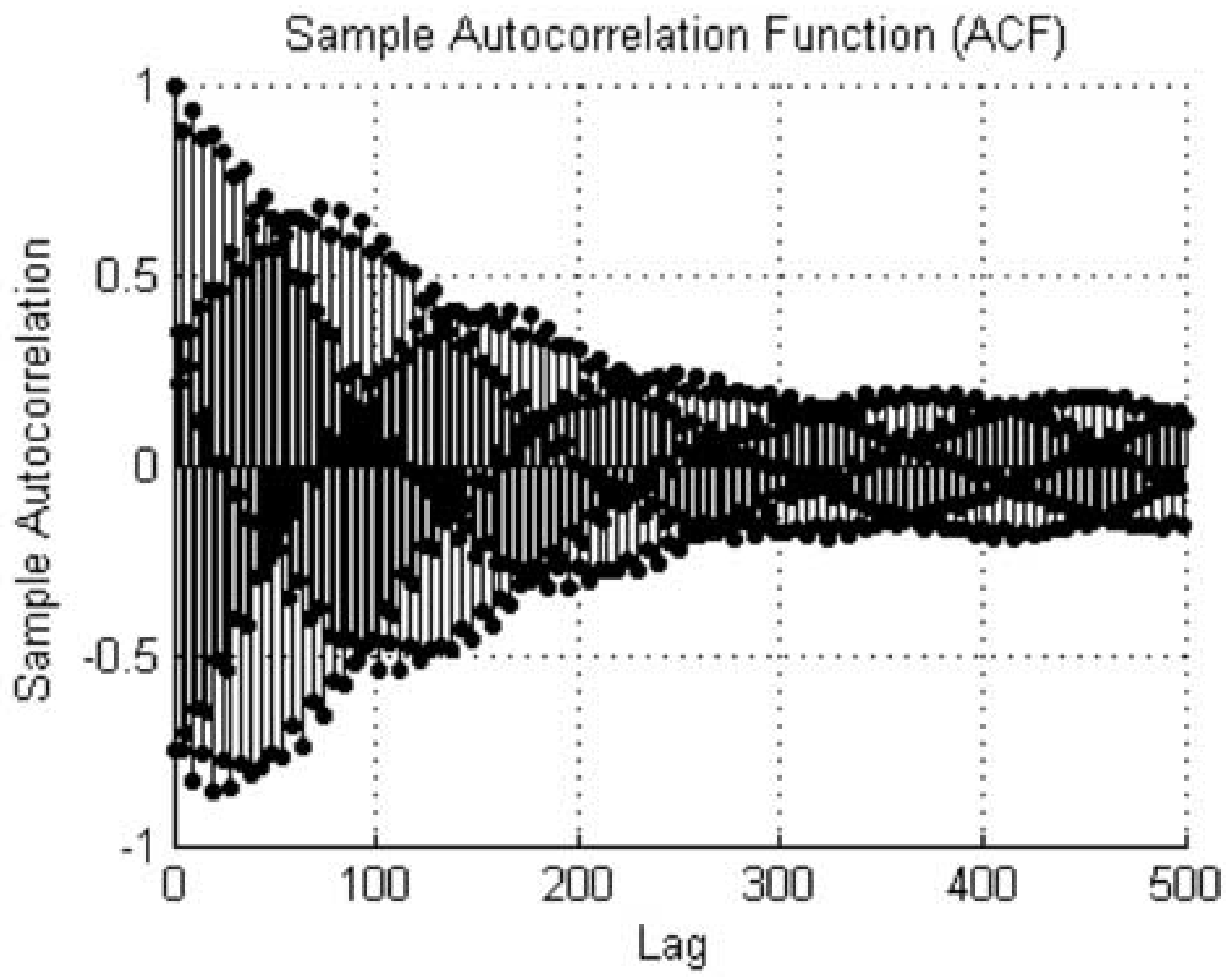


Figure $3 b$

Click here to download high resolution image

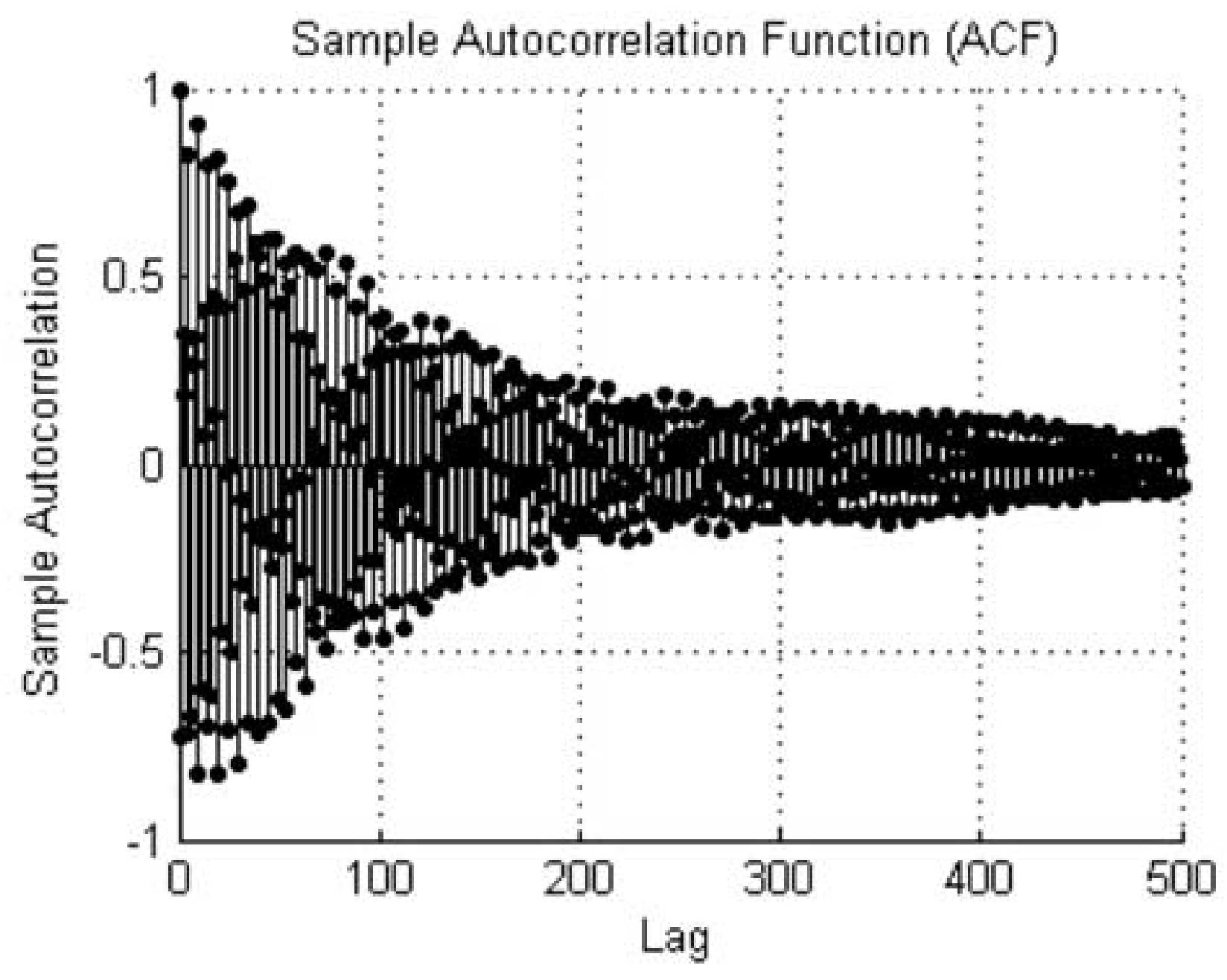

Sample Autocorrelation Function (ACF) 
Figure 4
Click here to download high resolution image

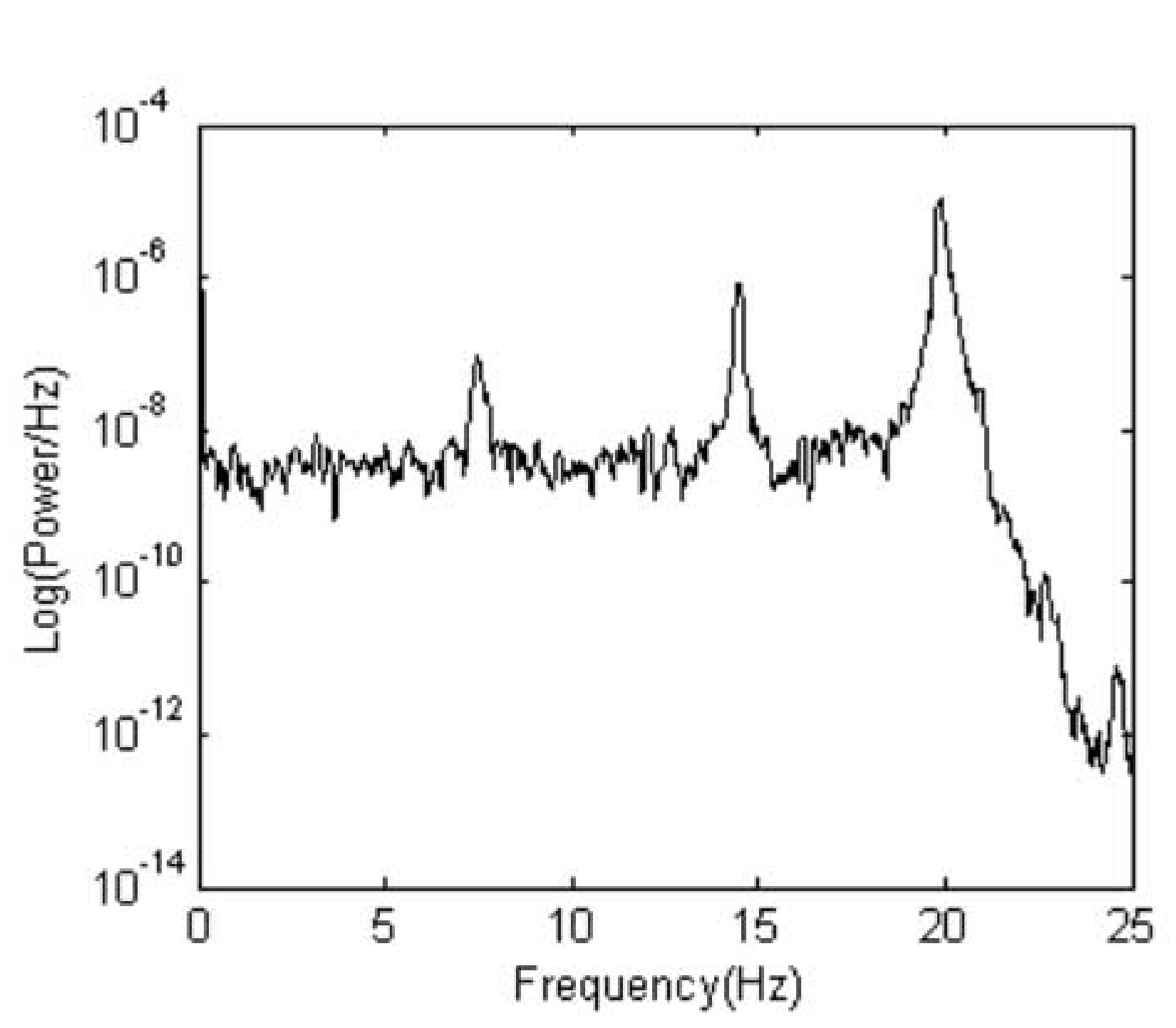

Click here to download high resolution image 
Figure $5 a$
Click here to download high resolution image

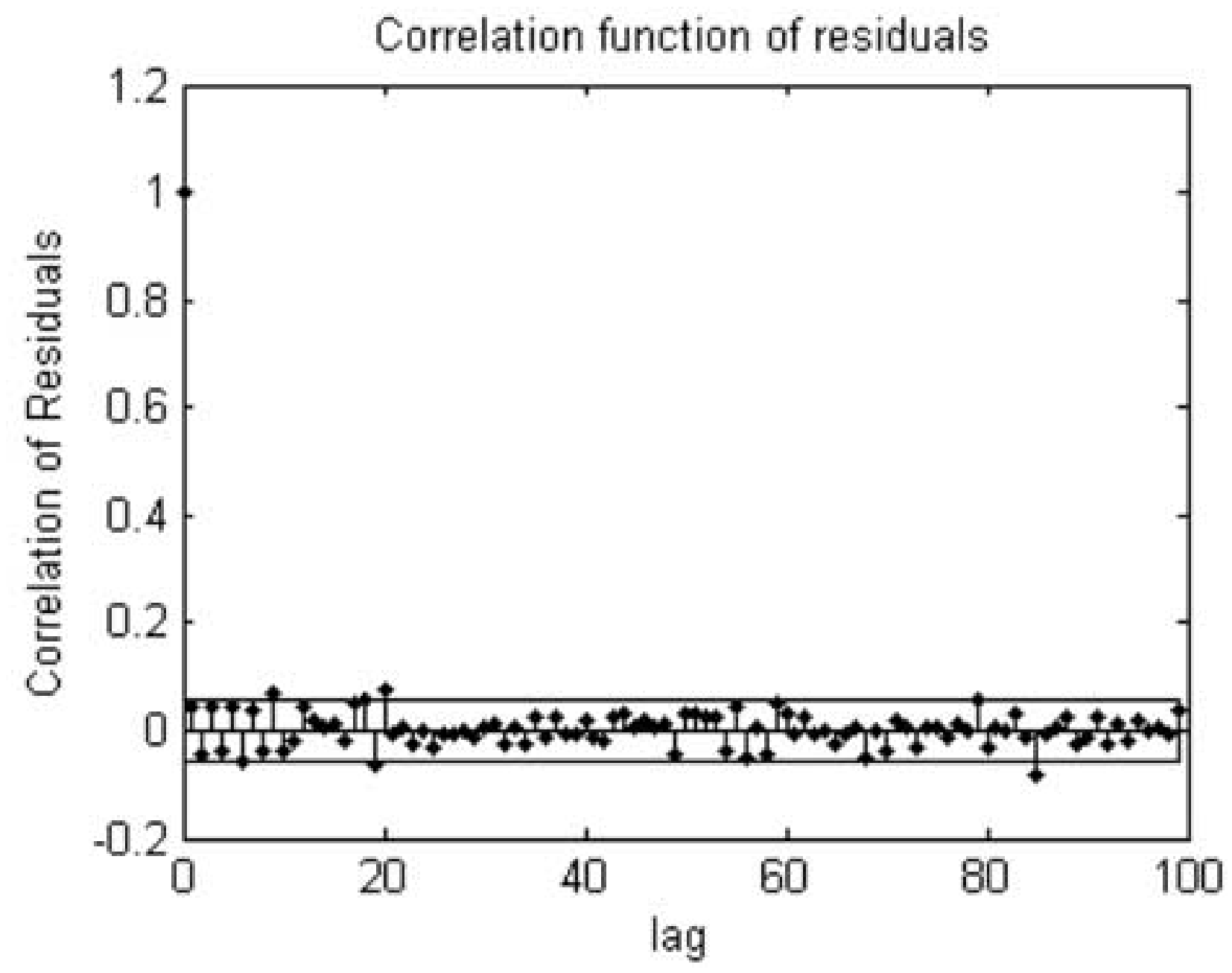

Correlation function of residuals 
Click here to download high resolution image
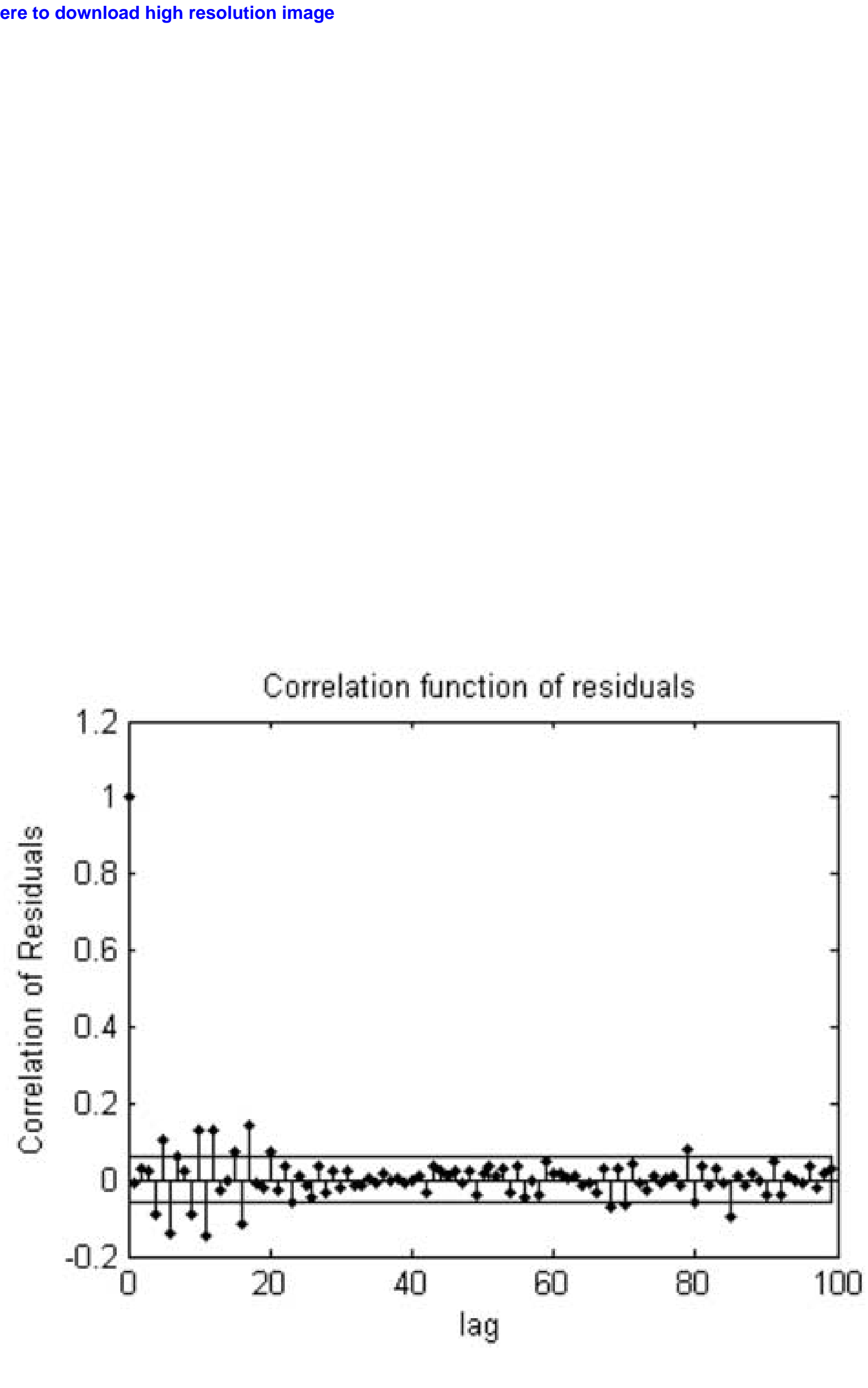


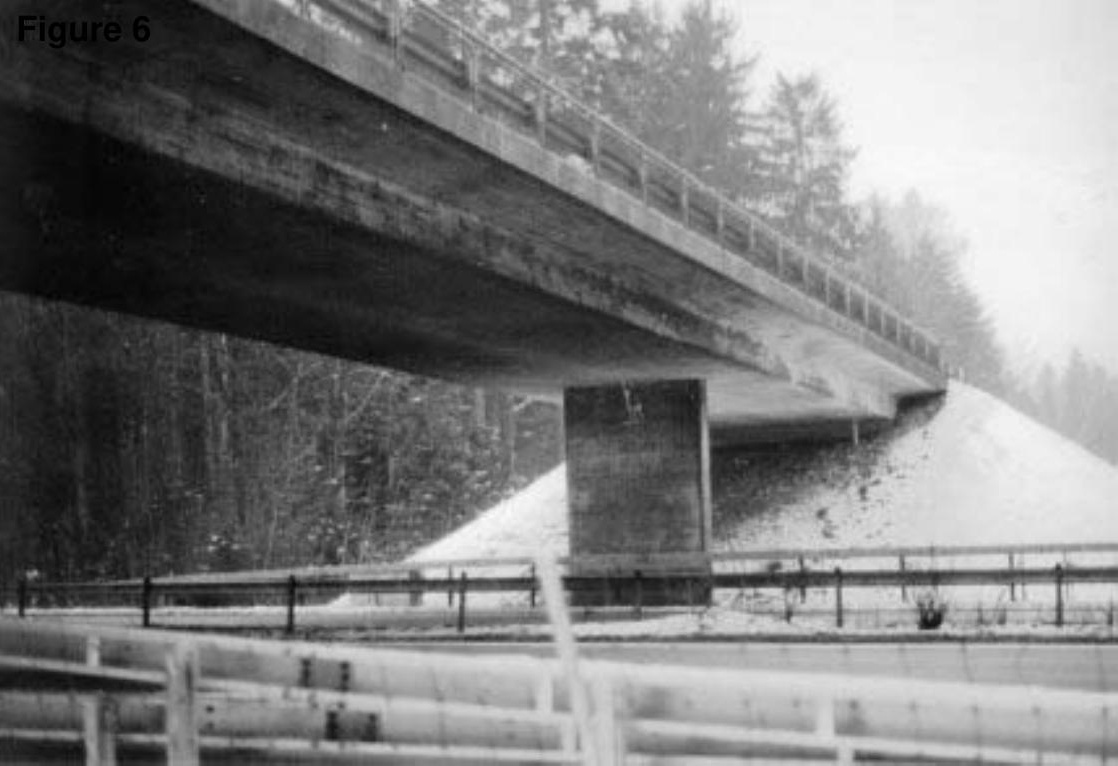


Figure 7

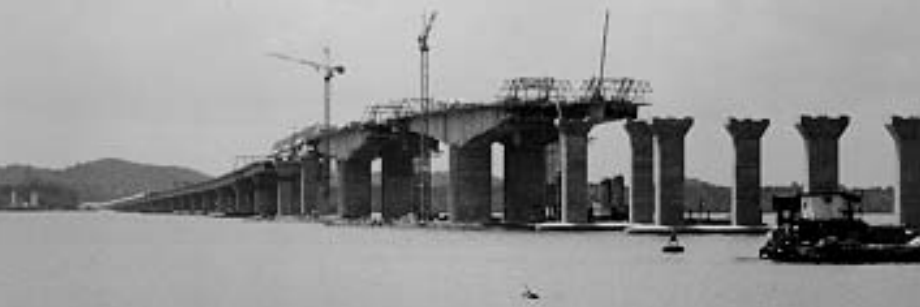


Figure $8 a$

Click here to download high resolution image

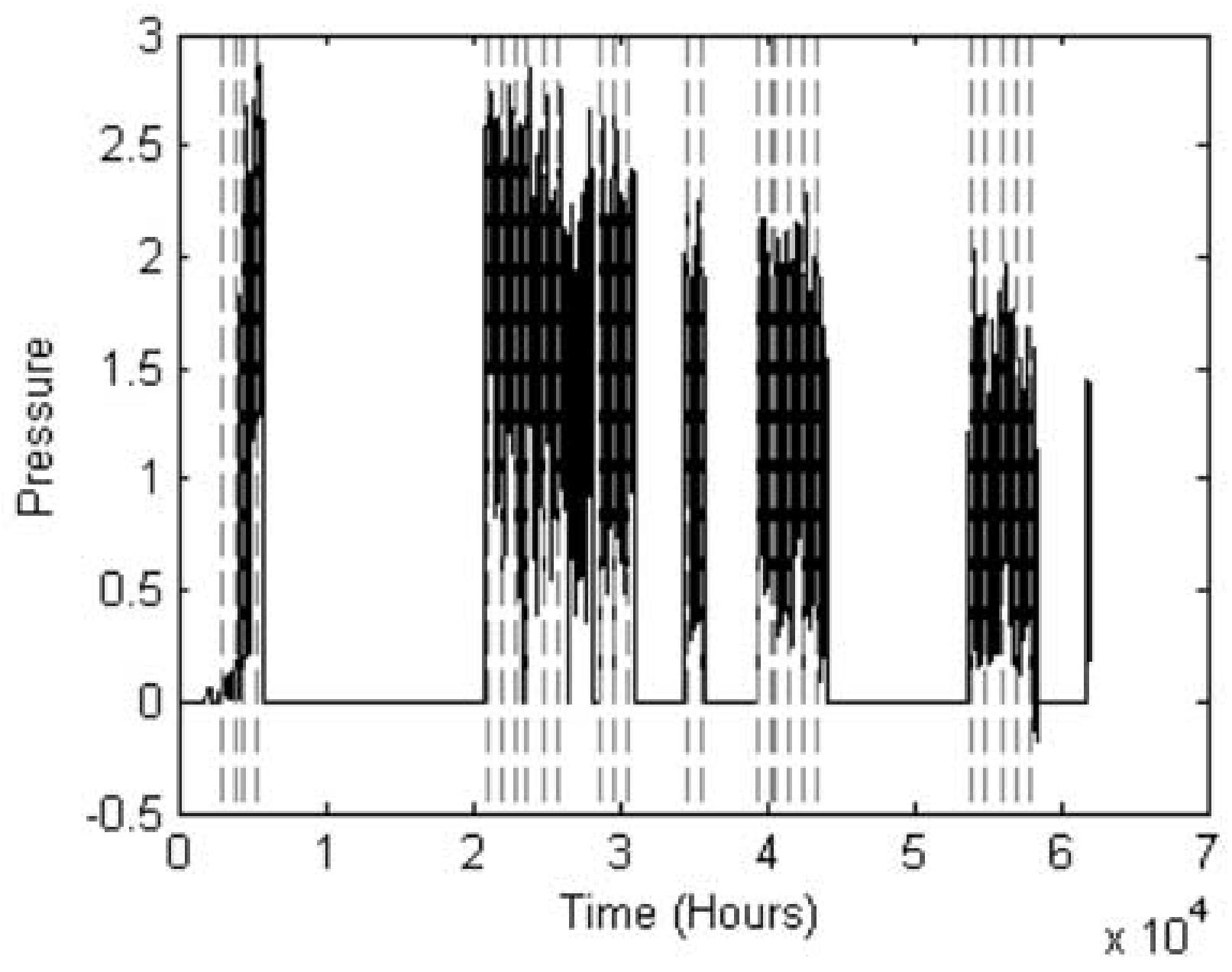




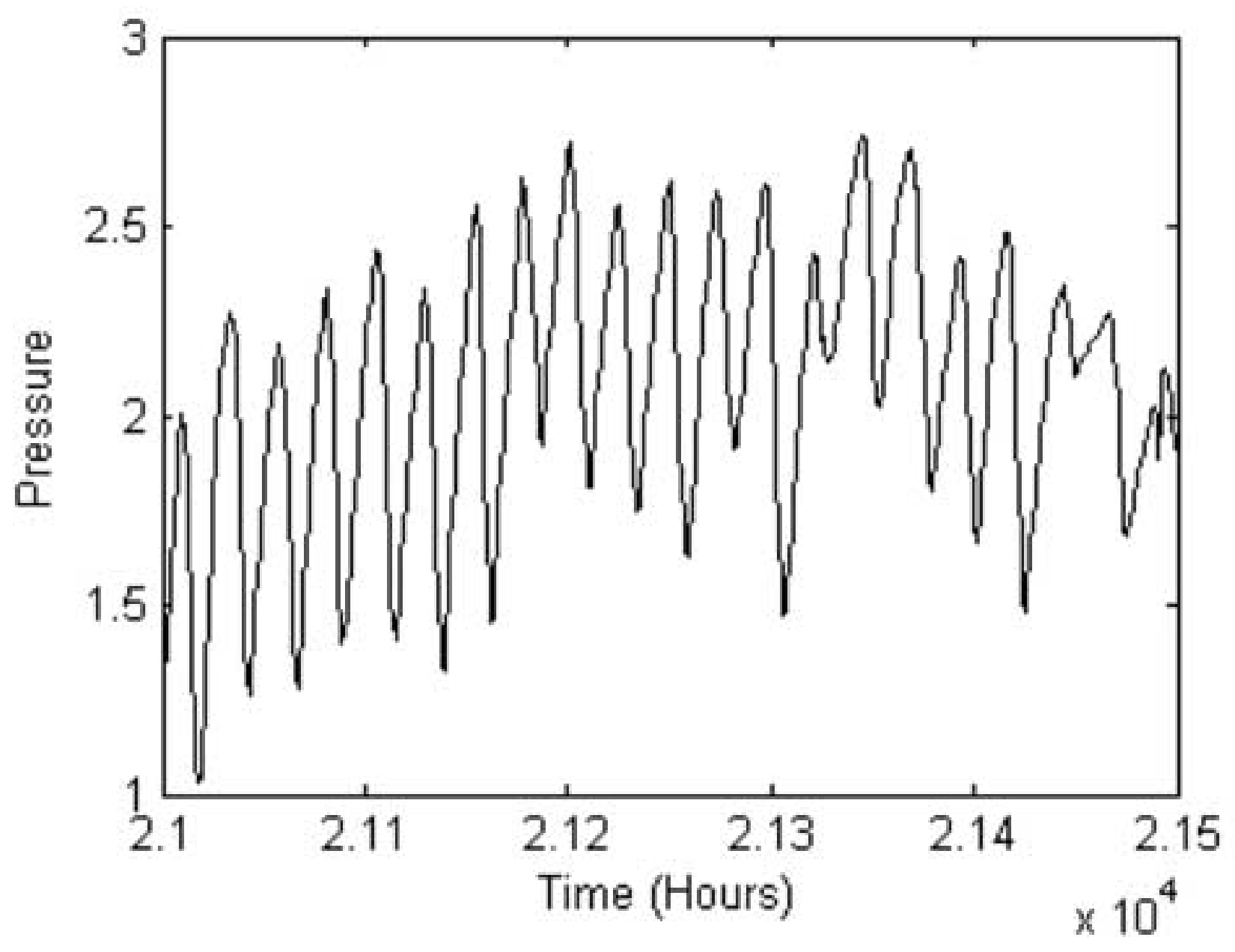


1 Fully Braced Configuration

2 Missing all East Side Braces

3 Removed Braces on all floors in one bay on SE corner

4 Removed Braces on 1st and 4th floors in one bay on SE corner

5 Removed Braces on 1st floor in one bay on SE corner

6 Removed Braces on all floors on East face, and 2nd floor braces on North face

7 All braced removed on all faces

8

Configuration 7, plus loosen bolts on all floors - both ends of beams on east face,

north side

Configuration 7, plus loosed bolts on floors 1 and 2 - both ends of beams on east face, north side 
Configuration Series Class Assigned

\begin{tabular}{|c|c|c|}
\hline \multirow[t]{3}{*}{1} & 1 & 1 \\
\hline & 2 & 1 \\
\hline & 3 & 1 \\
\hline \multirow[t]{3}{*}{2} & 1 & 2 \\
\hline & 2 & 2 \\
\hline & 3 & 3 \\
\hline \multirow[t]{3}{*}{3} & 1 & 4 \\
\hline & 2 & 4 \\
\hline & 3 & 5 \\
\hline \multirow[t]{3}{*}{4} & 1 & 6 \\
\hline & 2 & 6 \\
\hline & 3 & 6 \\
\hline \multirow[t]{3}{*}{5} & 1 & 7 \\
\hline & 2 & 7 \\
\hline & 3 & 7 \\
\hline \multirow[t]{3}{*}{6} & 1 & 8 \\
\hline & 2 & 8 \\
\hline & 3 & 9 \\
\hline \multirow[t]{3}{*}{7} & 1 & 10 \\
\hline & 2 & 10 \\
\hline & 3 & 10 \\
\hline \multirow[t]{3}{*}{8} & 1 & 11 \\
\hline & 2 & 11 \\
\hline & 3 & 11 \\
\hline \multirow[t]{3}{*}{9} & 1 & 12 \\
\hline & 2 & 13 \\
\hline & 3 & 13 \\
\hline
\end{tabular}




$\begin{array}{lll}\text { PDT No. } & \text { Scenario } & \text { Extent/Progression } \\ 1 & 1^{\text {st }} \text { Reference } & \text { No Damage } \\ 2 & 2^{\text {nd }} \text { Reference } & \begin{array}{l}\text { 'No Damage', Koppigen } \\ \text { Pier installed }\end{array} \\ 3 & & 20 \mathrm{~mm} \\ 4 & 1^{\text {st }} \text { Settlement of Pier } & 40 \mathrm{~mm} \\ 5 & 2^{\text {nd }} \text { Settlement of Pier } & 80 \mathrm{~mm} \\ 6 & 3^{\text {rd }} \text { Settlement of Pier } & 95 \mathrm{~mm} \\ 7 & 4^{\text {th }} \text { Settlement of Pier } & 15 \mathrm{~mm} \\ 8 & \text { Tilt of Foundation } & \text { 'No Damage' } \\ 9 & 3^{\text {rd }} \text { Reference } & 12 \mathrm{~m} \\ 10 & 1^{\text {st }} \text { Spalling of Concrete } & 24 \mathrm{~m} \\ 11 & 2^{\text {nd }} \text { Spalling of Concrete } & 1 \mathrm{~m} \\ 12 & \text { Landslide } & 1 \text { column } \\ 13 & \text { Failure of Concrete Hinges } & \\ 14 & 1^{\text {st }} \text { Failure of Anchor Heads } & 2 \text { heads } \\ 15 & 2^{\text {nd }} \text { Failure of Anchor Heads } & 4 \text { heads } \\ & 1^{\text {st }} \text { Failure of post } & 54 \text { wires or 2 tendons } \\ 16 & \text { Tensioning Wires } & \\ & 2^{\text {nd }} \text { Failure of Post } & 100 \text { wires or } 4 \text { tendons } \\ & \text { Tensioning Wires } & \\ & & \end{array}$




$\begin{array}{cccc} & \text { Class } 1 & \text { Class } 2 & \text { Class3 } \\ \text { AR } & -0.7554 & -0.7510 & -0.3721 \\ \mathrm{MA}_{\mathrm{s}} & -0.8559 & -0.8507 & -0.8479\end{array}$

